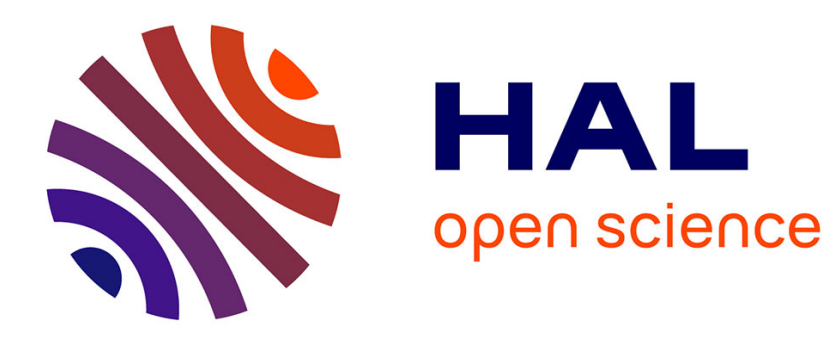

\title{
Routing into parallel collision channels
}

Mandar Datar, Eitan Altman, Ghilas Ferrat

\section{To cite this version:}

Mandar Datar, Eitan Altman, Ghilas Ferrat. Routing into parallel collision channels. MAMA 2020

- Workshop in conjunction with ACM/Sigmetrics 2020, Jun 2020, Boston / Virtual, United States. hal-02931301

\section{HAL Id: hal-02931301 \\ https://hal.inria.fr/hal-02931301}

Submitted on 5 Sep 2020

HAL is a multi-disciplinary open access archive for the deposit and dissemination of scientific research documents, whether they are published or not. The documents may come from teaching and research institutions in France or abroad, or from public or private research centers.
L'archive ouverte pluridisciplinaire HAL, est destinée au dépôt et à la diffusion de documents scientifiques de niveau recherche, publiés ou non, émanant des établissements d'enseignement et de recherche français ou étrangers, des laboratoires publics ou privés. 


\section{Routing into parallel collision channels}

\author{
Mandar Datar, \\ INRIA Sophia Antipolis - \\ Méditerranée \\ mandar.datar@inria.fr
}

\author{
Eitan Altman, \\ INRIA Sophia Antipolis - \\ Méditerranée \\ eitan.altman@inria.fr
}

\author{
Ghilas Ferrat, \\ INRIA Sophia Antipolis - \\ Méditerranée \\ ghilas.ferrat@inria.fr
}

\begin{abstract}
We study a Medium Access game modeled as a splittable atomic routing game in a parallel link topology. Each player has to decide how to split her traffic among the links. We take the expected loss probability of a player as her cost and consider various loss scenarios: 1) the $M / M / 1 / 1$ queue in which an arrival that finds another packet in service is lost, 2) Losses occur as in 1, except that if arrival occurs when another packet is served, then both are lost. We furthermore assume that the packet in service is aborted if there was a collision. 3) Like 2, but the packet in service is not aborted. We study the existence and uniqueness of equilibrium under these three types of losses.
\end{abstract}

\section{Keywords}

Routing games, Loss probabilities, Parallel links

\section{INTRODUCTION}

Traditionally tolls and delays have been considered as the performance measures in routing games. We have been studying other cost criteria related to loss of packets, see, e.g., [3]. In this paper, we study three different types of collisions and compare their equilibrium behavior. We assume that service times are independent exponentially distributed, and each player has as input an independent Poisson arrival point process. We obtain the best response and the equilibria in some cases and study their properties.

\section{MODEL AND PROBLEM FORMULATION}

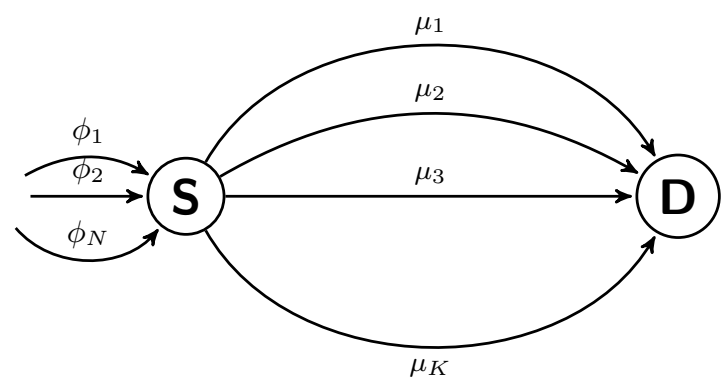

We consider a set of users $\mathcal{N}=\{1,2 \ldots N\}$ which shares the set of parallel links $\mathcal{K}=\{1,2 \ldots K\}$. Service times at each link (server) $k \in \mathcal{K}$ are independent and exponentially distributed with mean $\mu_{k}$. The arrival process to each user $i \in \mathcal{N}$ is an independent Poisson process with rate $\phi_{i}$. User $i$ splits its demand into $K$ independent processes with rates $\lambda^{i}:=\left\{\lambda_{k}^{i}, k=1, \ldots, K\right\}$, using independent trials in which packet arriving at player $i$ is forwarded to link $k$ with probability $p_{k}^{i}=\lambda_{k}^{i} / \phi_{i}$. If during the service time of a packet, another one arrives then, we consider the three cases of loss

1. the arriving packet is lost

2. both arriving and served packets are lost. Just after the collision, transmission and service are aborted, and an idle period (exponentially distributed with paramter ג) starts.

3. both the packets get corrupted; however, the packet which was in service continues to be in service; the fact that it is corrupt is discovered only when it arrives at the destination.

Our contribution is in establishing sufficient conditions for the existence and uniqueness of a Nash equilibrium as well as for the DSC (and hence of the scalable equilibrium). First, we model the loss channels and compute the loss probabilities of the players as a function of their policies. We then study in Section 4 conditions for DSC. We end with a concluding section.

\section{MODELLING AND COMPUTING LOSS PROBABILITIES}

Now we find a loss rate for the users; we define renewal cycle $C=I+B$, the period in between the two successive packet transmission or collision events. Where $I$ is the idle period, and $B$ is the busy period.

For case 1, the busy period is exponentially distributed with parameter $\mu_{k}$. Indeed, B is unchanged if we replace the served packet with a loss of an arrival packet. The loss probability is $\frac{\mathbb{E}[B]}{\mathbb{E}[I]+\mathbb{E}[B]}$ by Pasta (Poisson Arrivals See Time Averages) property. where $\mathbb{E}[I]=\frac{1}{\lambda_{k}}, \mathbb{E}[B]=\frac{1}{\mu_{k}}$.

$$
P_{k}(\lambda)=\frac{\sum_{j=1}^{N} \lambda_{k}^{j}}{\sum_{j=1}^{N} \lambda_{k}^{j}+\mu_{k}}
$$

where $\lambda$ is the multipolicy, i.e. the set whose $i, k$ element is $\lambda_{k}^{i}$. Thus the total average loss rate of player $i$ is

$$
J^{i}(\lambda)=\sum_{k} \frac{\left(\lambda_{k}^{i}\right) \sum_{j=1}^{N} \lambda_{k}^{j}}{\sum_{j=1}^{N} \lambda_{k}^{j}+\mu_{k}}
$$


For the case 2 , we have $\mathbb{E}[I]=\frac{1}{\lambda}$ and $\mathbb{E}[B]=\frac{1}{\lambda_{k}+\mu_{k}}$. and the probability of finding a packet in service on arrival is

$$
Q=\frac{\mathbb{E}[B]}{\mathbb{E}[I]+\mathbb{E}[B]}=\frac{\frac{1}{\lambda_{k}+\mu_{k}}}{\frac{1}{\lambda_{k}+\mu_{k}}+\frac{1}{\lambda_{k}}}
$$

The probability of a loss is

$$
P_{k}(\lambda)=Q+(1-Q) \frac{\lambda_{k}}{\lambda_{k}+\mu_{k}}
$$

The total average loss rate of player $i$ in the case 2 is

$$
J^{i}(\lambda)=\sum_{k} \frac{2\left(\lambda_{k}^{i}\right) \sum_{j=1}^{N} \lambda_{k}^{j}}{2 \sum_{j=1}^{N} \lambda_{k}^{j}+\mu_{k}}
$$

In case 3 , whenever there is the arrival of a packet, and it finds another packet in service, both the packets get corrupted however the packet which was in service continues to be in service on the arrival of any packet, probability that it finds server busy is $P=\frac{\lambda_{k}}{\lambda_{k}+\mu_{k}}$ therefore packet loss probability is $p+(1-p) \frac{\lambda_{k}}{\lambda_{k}+\mu_{k}}$. Where $(1-p) \frac{\lambda_{k}}{\lambda_{k}+\mu_{k}}$ is the probability of packet joins the service and gets corrupted due to the next arrival. The Packet loss rate for player $i$

$$
J^{i}\left(\lambda_{k}, \lambda_{k}^{i}\right)=\left(\frac{\lambda_{k}}{\lambda_{k}+\mu_{k}}+\frac{\mu_{k}}{\lambda_{k}+\mu_{k}} \frac{\lambda_{k}}{\lambda_{k}+\mu_{k}}\right) \lambda_{k}^{i}
$$

Total average loss

$$
J^{i}\left(\lambda_{k}, \lambda_{k}^{i}\right)=\sum_{k}\left(\frac{\lambda_{k}}{\lambda_{k}+\mu_{k}}+\frac{\mu_{k}}{\lambda_{k}+\mu_{k}} \frac{\lambda_{k}}{\lambda_{k}+\mu_{k}}\right) \lambda_{k}^{i}
$$

The goal of each user is to minimize its total job drop rate. Since the job drop rate incurred by the user depends on the decisions made by other users and users are selfish, we are faced with a noncooperative game.

we define the non-cooperative game $\mathcal{G}$ as follows:

- Player set: The set of users $\mathcal{N}=\{1,2 \ldots N\}$ which shares the set of parallel links

- Strategy: It is defined as the amount of flow $\lambda^{i}=$ $\left[\lambda_{1}^{i} \ldots \lambda_{K}^{i}\right]$ to be routed to the each link strategy set is $\left\{\lambda^{i} \mid \sum_{k} \lambda_{k}^{i}=\phi_{i}\right\}$

- Cost: The cost of each player $i$ is equal to loss rate $J^{i}$

For this game, we consider the standard notion of a Nash equilibrium,

Definition 1. A multistrategy $\lambda^{*}=\left(\lambda^{i^{*}}, \ldots, \lambda^{N^{*}}\right)$ is called a Nash Equilibrium of the game if

$$
\forall i \in \mathcal{N}, J^{i}\left(\lambda^{i^{*}}, \lambda^{-i^{*}}\right) \leq J^{i}\left(\lambda^{i}, \lambda^{-i^{*}}\right)
$$

Here, $\left(\lambda^{i}, \lambda^{-i^{*}}\right)$ refers to the strategy vector which $i^{\text {th }}$ element equals $\lambda$ and all other elements equal $\lambda^{j *}$ (for any $j \neq i$ )

We show the existence of equilibrium, for we first examine the convexity of loss rate. The loss rate function for case 1 and case 2 only differ in their coefficient. All the results of case 1 also holds for the case 2 consider $\frac{\partial^{2} J_{k}^{i}}{\partial \lambda_{k}^{i 2}}$

For case 1

$$
\frac{\partial^{2} J_{k}^{i}}{\partial \lambda_{k}^{i^{2}}}=\frac{\mu_{k}\left(\sum_{j=1, j \neq i}^{N} \lambda_{k}^{j}\right)}{\left(\sum_{j} \lambda_{m}^{j}+\mu_{k}\right)^{3}}
$$

For case 3

$$
\frac{\partial^{2} J_{k}^{i}}{\partial \lambda_{k}^{i}}=\frac{-6 \mu_{k}{ }^{2}}{\left(\lambda_{k}+\mu_{k}\right)^{4}} \lambda_{k}^{i}+\frac{4 \mu_{k}{ }^{2}}{\left(\lambda_{k}+\mu_{k}\right)^{3}}
$$

For case $1, \frac{\partial^{2} J_{k}^{i}}{\partial \lambda_{k}^{i}}>0$, while

for case $3 \frac{\partial^{2} J_{k}^{i}}{\partial \lambda_{k}^{i 2}}>0$ If $\lambda_{k}^{i}<2 \sum_{j \neq i} \lambda_{k}^{j}+2 \mu_{k}$

Result 1 For case 1 , the job drop rate $J^{i}\left(\lambda^{i}\right.$, .) is convex function of the strategy of player $i$ and the strategy set is compact, hence a Nash equilibrium exists [1]. Similarly, For case 3 , If $\lambda_{k}^{i}<2 \sum_{j \neq i} \lambda_{k}^{j}+2 \mu_{k}$ then Nash equilibrium exists. In the next section, we will explore the properties of nash equilibrium; particularly, we will see whether the equilibrium is unique.

\section{DIAGONAL STRICT CONVEXITY}

To show the uniqueness of nash equilibrium, we first recall the tool, introduced by Rosen [1] called strict Diagonal concavity. In the seminal work of Rosen, the term Diagonal strict concavity was used. In this paper, the goal of players is to minimize the loss rate function (convex); hence we use the term Diagonal strict convexity.

DEFINITION 2. For a vector of real non-negative numbers $r$, define

$$
\sigma(\lambda, r)=\sum_{i=1}^{N} r_{i} J^{i}(\lambda)
$$

$\sigma$ is called diagonally strict convex (DSC) for a given $r$ if for every distinct $\hat{\lambda}$ and $\bar{\lambda}$,

$$
\sum_{i=1}^{N} r_{i}\left(\bar{\lambda}^{i}-\hat{\lambda}^{i}\right)^{\prime}\left[\left.\frac{\partial J^{i}}{\partial \lambda^{i}}\right|_{\bar{\lambda}}-\left.\frac{\partial J^{i}}{\partial \lambda^{i}}\right|_{\hat{\lambda}}\right]>0
$$

Theorem 1. (Theorem 2 from [1]) Assume that $\sigma$ is diagonally strict concave for some $r>0$. Then there exists a unique Nash equilibrium.

Next, recall the sufficient conditions for Diagonal Strict Concavity (DSC). The $\sigma$ is diagonally strict convex if $\left[G+G^{\prime}\right]$ is positive definite where (DSC is a property that generalizes convexity to a setting of games. It implies the uniqueness of the equilibrium and provides convergence of to that equilibrium, see [1]). Where the $G$ is pseudo-Jacobin of $\left[\frac{\partial J^{1}}{\partial \lambda^{1}} \cdots \frac{\partial J^{K}}{\partial \lambda^{K}}\right]$ and $(i, j)^{t h}$ element of $G$ is

$$
G_{i, j}=\frac{\partial}{\partial \lambda^{j}}\left(\frac{\partial}{\partial \lambda^{i}} J^{i}\right)
$$

$\left[G+G^{\prime}\right]$ can be calculated by finding $\left[G^{k}+G^{\prime k}\right]$ for each link $k$ separately and then writing as

$$
\left[G+G^{\prime}\right]=\operatorname{Diag}\left\{\left[G^{1}+G^{\prime 1}\right], \ldots,\left[G^{K}+G^{\prime K}\right]\right\}
$$

Now we calculate $\left[G^{k}+G^{\prime k}\right]$ for case 1 ,

$$
G_{i, j}^{k}= \begin{cases}\frac{\mu_{k}\left(\sum_{j=1, j \neq i}^{N} \lambda_{k}^{j}\right)}{\left(\sum_{j} \lambda_{m}^{j}+\mu_{k}\right)^{3}} & \text { if } i=i \\ \frac{-\mu_{k}\left(\lambda_{k}^{i}-\sum_{j \neq i} \lambda_{k}^{j}\right)}{\left(\sum_{j} \lambda_{m}^{j}+\mu_{k}\right)^{3}} & \text { if } i=j\end{cases}
$$


for case 3

$$
G_{i, j}^{k}= \begin{cases}\frac{-6 \mu_{k}{ }^{2}}{\left(\lambda_{k}+\mu_{k}\right)^{4}} \lambda_{k}^{i}+\frac{4 \mu_{k}{ }^{2}}{\left(\lambda_{k}+\mu_{k}\right)^{3}} & \text { if } i=i \\ \frac{-6 \mu_{k}}{\left(\lambda_{k}+\mu_{k}\right)^{4}} \lambda_{k}^{i}+\frac{2 \mu_{k}}{\left(\lambda_{k}+\mu_{k}\right)^{3}} & \text { if } i=j\end{cases}
$$

We shall prove next that DSC holds for any number K of parallel links and 2 players. This implies the uniqueness of the Nash equilibrium [1]. For the case 1, we calculate the above matrix's determinant for two users and single link $k$, which is

$$
\frac{16\left(\frac{3}{4} \mu_{k}^{2}+\left(\lambda_{k}^{1}+\lambda_{k}^{2}\right) \mu_{k}+\lambda_{k}^{1} \lambda_{k}^{2}\right) \mu_{k}^{2}}{\left(\lambda_{k}^{1}+\lambda_{k}^{2}+\mu_{k}\right)^{6}}>0
$$

Therefore $\left[G^{k}+G^{\prime k}\right]$ is positive definite for each $k$ Similarly, for the case 3 , we find the determinant of $\left[G^{k}+G^{\prime}\right]$

$$
-36 \frac{\mu_{k}{ }^{4}\left(-4 / 3\left(\mu_{k}{ }^{2}+\left(\lambda_{k}^{1}-\lambda_{k}^{2}\right) \mu_{k}\right)+\left(\lambda_{k}^{1}-\lambda_{k}^{2}\right)^{2}\right)}{\left(\lambda_{k}^{1}+\lambda_{k}^{2}+\mu_{k}\right)^{8}}
$$

The determinant of matrix shows, it is not necessary that $\left[G^{k}+G^{\prime k}\right]$ is always positive definite, hence for the case 3, nothing can be said about the uniqueness in general.

Now to prove uniqueness of equilibrium, we use the method discussed in [2], we write the loss rate as function of $\lambda_{k}^{i}$ and $\lambda_{k}, J_{k}^{i}\left(\lambda_{k}^{i}, \lambda_{k}\right)$ and let define $H_{k}^{i}=\frac{\partial J_{k}^{i}}{\partial \lambda_{k}^{i}}$ then For case 1 ,

$$
\begin{aligned}
& H_{k}^{i}\left(\lambda_{k}^{i}, \lambda_{k}\right)=\frac{\mu_{k} \lambda_{k}^{i}}{\left(\lambda_{k}+\mu_{k}\right)^{2}}+\frac{\lambda_{k}}{\lambda_{k}+\mu_{k}} \\
& \frac{\partial H_{k}^{i}\left(\lambda_{k}^{i}, \lambda_{k}\right)}{\partial \lambda_{k}}=\frac{\mu_{k}\left(\lambda_{k}^{-i}-\lambda_{k}^{i}+\mu_{k}\right)}{\left(\lambda_{k}+\mu_{k}\right)^{3}}
\end{aligned}
$$

from above two equations we have following observations

1. $J_{k}^{i}\left(\lambda_{k}^{i}, \lambda_{k}\right)$ is increasing and convex in $\lambda_{k}^{i}$

2. $J_{k}^{i}\left(\lambda_{k}^{i}, \lambda_{k}\right)$ is increasing in $\lambda_{k}$

3. $H_{k}^{i}\left(\lambda_{k}^{i}, \lambda_{k}\right)$ is increasing in $\lambda_{k}^{i}$

4. $H_{k}^{i}\left(\lambda_{k}^{i}, \lambda_{k}\right)$ is increasing in $\lambda_{k}$ if $\left(\lambda_{k}^{-i}-\lambda_{k}^{i}+\mu_{k}\right)>0$

Similarly for Case 3, we have

$$
H_{k}^{i}\left(\lambda_{k}^{i}, \lambda_{k}\right)=\frac{2 \mu_{k}{ }^{2}}{\left(\lambda_{k}+\mu_{k}\right)^{3}} \lambda_{k}^{i}+\frac{\lambda_{k}}{\lambda_{k}+\mu_{k}}\left(1+\frac{\mu_{k}}{\lambda_{k}+\mu_{k}}\right)
$$

differentiating $H_{k}^{i}\left(\lambda_{k}^{i}, \lambda_{k}\right)$ with respect to $\lambda_{k}$

$$
\begin{aligned}
\frac{\partial H_{k}^{i}\left(\lambda_{k}^{i}, \lambda_{k}\right)}{\partial \lambda_{k}} & =\frac{-6 \mu_{k}{ }^{2}}{\left(\lambda_{k}+\mu_{k}\right)^{4}} \lambda_{k}^{i}+\frac{4 \mu_{k}{ }^{2}}{\left(\lambda_{k}+\mu_{k}\right)^{3}} \\
& =\left(-6 \lambda_{k}^{i}+4 \lambda_{k}+4 \mu_{k}\right) \frac{\mu_{k}{ }^{2}}{\left(\lambda_{k}+\mu_{k}\right)^{4}}
\end{aligned}
$$

If $\lambda_{k}^{i}<2 \sum_{j \neq i} \lambda_{k}^{j}+2 \mu_{k}$ then $\frac{\partial H_{k}^{i}\left(\lambda_{k}^{i}, \lambda_{k}\right)}{\partial \lambda_{k}}>0$ and following statements hold

1. $J_{k}^{i}\left(\lambda_{k}^{i}, \lambda_{k}\right)$ is increasing and convex in $\lambda_{k}^{i}$

2. $J_{k}^{i}\left(\lambda_{k}^{i}, \lambda_{k}\right)$ is increasing in $\lambda_{k}$

3. $H_{k}^{i}\left(\lambda_{k}^{i}, \lambda_{k}\right)$ is increasing in $\lambda_{k}^{i}$
4. $H_{k}^{i}\left(\lambda_{k}^{i}, \lambda_{k}\right)$ is increasing in $\lambda_{k}$

THEOREM 2. For case 1 , If $\left(\lambda_{k}^{-i}-\lambda_{k}^{i}+\mu_{k}\right)>0$ for each link $k$ and user $i$ then there esxist unique Nash equilibrium to routing game $\mathcal{G}$.

For case 3, If $\lambda_{k}^{i}<2 \sum_{j \neq i} \lambda_{k}^{j}+2 \mu_{k}$ for each link $k$ and user $i$ then there esxist unique Nash equilibrium to routing game $\mathcal{G}$.

Proof. If for case $1,\left(\lambda_{k}^{-i}-\lambda_{k}^{i}+\mu_{k}\right)>0$ and case 3 $\lambda_{k}^{i}<2 \sum_{j \neq i} \lambda_{k}^{j}+2 \mu_{k}$ then assumptions(statements 1 to 4 ) $A 1-A 3$ from [2] are satisfied, and then uniqueness follows from [Theorem 1 [2]]

\section{CONCLUSION AND FUTURE WORK}

Orda, Rom, and Shimkin show in their seminal paper [2] that holds in the above game with parallel links for two players and two parallel links only in light load. This was proved for queueing delay type cost. We showed that this result extends to any number of links and for 2 or 3 players when considering our average loss criterion instead of the average delay criterion.

In present work, we have considered the orthogonal constraint, a constraint on the actions of a player is said to be orthogonal if its satisfaction by the player does not depend on the actions of other players. We note that the nonnegativity of flows, as well as total demand constraints, are orthogonal constraints. Rosen [1] introduced the concept of coupled constraints in which the set of actions available to the players is a common convex subset of actions. This requires some type of cooperation between players to satisfy the coupled constraints.

Normalized equilibrium: At equilibrium, each player's best response can be derived from the minimization of a cost function. According to the Karush Kuhn Tucker Theorem, A player's best response can be obtained by Lagrange relaxation, where the cost to be minimized replaced by a Lagrangian for some Lagrange multipliers. These multipliers can be considered as pricing. In general, these prices need not be unique. Furthermore, prices may differ from one player to another. In case prices are unique and independent of the player, then the equilibrium and prices are called scalable equilibrium. Rosen shows that DSC implies the existence and uniqueness of the scalable equilibrium. Other consequences of the diagonal strict concavity are algorithms that allow for gradient-type learning algorithms to converge to the unique Nash equilibrium. Such an algorithm is provided in [1].

\section{REFERENCES}

[1] J. B. Rosen: Existence and Uniqueness of Equilibrium Points for Concave N-Person Games. Econometrica Vol. 33, No. 3 (Jul., 1965), pp. 520-534

[2] A. Orda, R. Rom and N. Shimkin:Competitive routing in multiuser communication networks. IEEE/ACM Transactions on Networking, vol. 1, no. 5, pp. 510-521, Oct. 1993.

[3] Eitan Altman, Joy Kuri, Rachid El-Azouzi. A routing game in networks with lossy links. 7th International Conference on NETwork Games COntrol and OPtimization (NETGCOOP 2014), Oct 2014, Trento, Italy. hal-01066453 